This item was submitted to Loughborough's Research Repository by the author.

Items in Figshare are protected by copyright, with all rights reserved, unless otherwise indicated.

\title{
Computational study of a battle damaged finite aspect ratio wing
}

PLEASE CITE THE PUBLISHED VERSION

PUBLISHER

(C) Peter Render. Published by the American Institute of Aeronautics and Astronautics, Inc., with permission.

\section{VERSION}

VoR (Version of Record)

LICENCE

CC BY-NC-ND 4.0

\section{REPOSITORY RECORD}

Yang, Zhiyin, Mujahid Samad-Suhaeb, and Peter M. Render. 2012. "Computational Study of a Battle Damaged Finite Aspect Ratio Wing”. figshare. https://hdl.handle.net/2134/10068. 
This item was submitted to Loughborough's Institutional Repository (https://dspace.lboro.ac.uk/) by the author and is made available under the following Creative Commons Licence conditions.

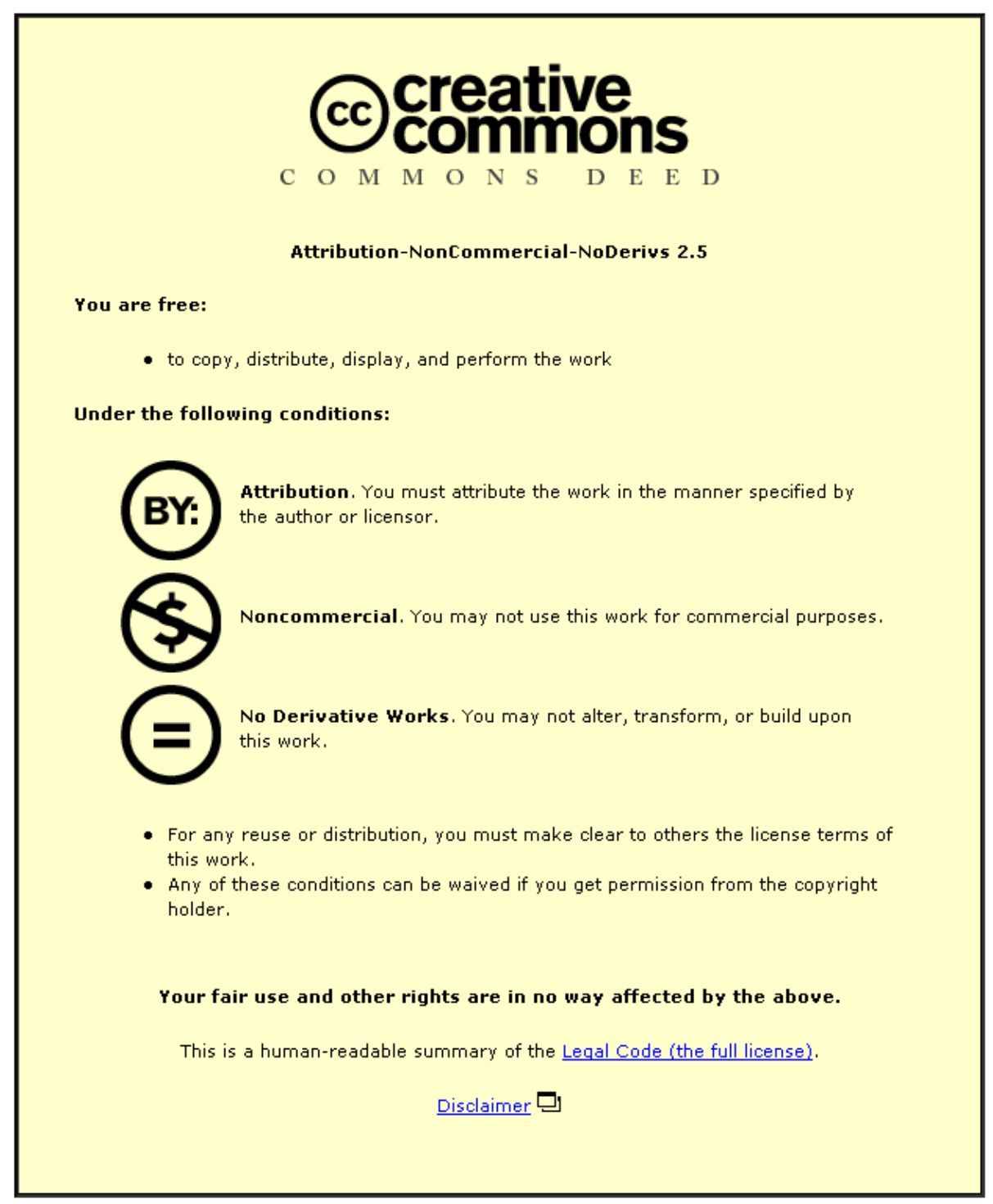

For the full text of this licence, please go to: http://creativecommons.org/licenses/by-nc-nd/2.5/ 


\title{
Computational Study of a Battle Damaged Finite Aspect Ratio Wing
}

\author{
Zhiyin Yang ${ }^{1}$, Mujahid Samaad-Suhaeb ${ }^{2}$ and Peter M. Render ${ }^{3}$ \\ Department of Aeronautical and Automotive Engineering, Loughborough University, \\ Loughborough, Leicestershire, England, LE11 3TU, United Kingdom
}

\begin{abstract}
Numerical studies have been carried out on a battle damaged NACA $64_{1}-412$ half wing of aspect ratio 8.2 at a Reynolds number of $5.5 \times 10^{5}$. The simulated gunfire damage was represented by a single hole with a diameter of 0.2 wing chord. The hole was centred at half chord and at spanwise locations of $450 \mathrm{~mm}$ and $650 \mathrm{~mm}$ from wing root. Computational Fluid Dynamics (CFD) results have been compared with experimental data and a general overall good agreement has been obtained, in the flow features introduced by the damage. Furthermore, the flow field inside the damage hole and in the region downstream of the jet have been analysed in more detail using CFD.
\end{abstract}

\section{Nomenclature}

$\begin{array}{ll}\mathrm{C} & =\text { wing chord } \\ \mathrm{C}_{\mathrm{p}} & =\text { static pressure coefficient } \\ \mathrm{X} & =\text { chordwise co-ordinate } \\ \mathrm{y} & =\text { vertical co-ordinate } \\ \mathrm{Z} & =\text { spanwise co-ordinate }\end{array}$

\section{Introduction}

$\mathrm{O}$ NE of the most important requirements for a military aircraft is its survivability in combat. Wing damage is of particular concern since the lift force is mainly generated by a wing. When an aircraft wing is damaged it is vital for the wing to maintain an acceptable level of aerodynamic efficiency to ensure a safe return and landing. However, previous research has mainly been concentrated on the structural behavior of a damaged wing and relatively little research has been carried out on the aerodynamics of battle-damaged finite-aspect-ratio wings, especially given that low-speed characteristics of a damaged wing are very relevant in determining if an aircraft will make a successful landing.

Despite a few early studies ${ }^{1,2,3,4}$ the lack of low speed data prompted the start of battle damage studies at Loughborough University. Irwin ${ }^{5}$ and Irwin \& Render $^{6}$ conducted a series of wind tunnel experiments in a low turbulence wind tunnel to determine how the aerodynamic characteristics of a two-dimensional NACA $64_{1}-412$ airfoil was influenced by the presence of simulated gunfire or missile damage. Using a wind tunnel balance, the increments in the drag, lift and pitching moment coefficients, relative to an undamaged airfoil, were determined for each of the damage cases. To assist in the analysis, balance measurements were supplemented by flow visualisation and surface static pressure readings.

Previous numerical studies on battle damaged wings are even more scarce, and Schemensky \& Howell ${ }^{7}$ are believed to have carried out the first numerical study in this area. They developed an empirically based computer program for determining the lift, drag and moment characteristics of aircraft that sustained nuclear damage. Few CFD studies in this area have been published so far. Numerical investigation of a damaged airfoil have been carried out by Rasi Marzabadi et al. ${ }^{8,9}$ and Saeedi et al. ${ }^{10}$. They have done a numerical study of a two-dimensional NACA $64_{1}-412$ airfoil with circle and star damage and also three repair configurations using the standard $\mathrm{k}-\varepsilon$ model.

\footnotetext{
${ }^{1}$ Currently: Reader, Dept. of Engineering and Design, Sussex University, Brighton, BN1 9RH, England

${ }^{2}$ Currently: Managing Director, Jakarta Aerospace, Plaza Aminta Building, Jl.TB.Simatupang Kav.10 - Jakarta Selatan 12310, Indonesia

${ }^{3}$ Senior Lecturer. Senior Member AIAA.
} 
This paper describes a numerical study of a NACA $64_{1}-412$ half wing of aspect ratio 8.2 with circle damage. The main objectives of this paper are: 1). To assess the capability and accuracy of the Reynolds Averaged Navier-Stokes (RANS) approach for this kind of flow by comparing the CFD results with experimental data; 2). To analyse the flow field inside the damage hole and in the region downstream of the jet in more detail using CFD data.

\section{Numerical Methods}

The governing equations, called Navier-Stokes equations, are derived from the conservation laws for mass, momentum and energy. Under the RANS approach the governing equations are time or ensemble averaged. This averaging process leads to additional terms called Reynolds stresses and hence a turbulence model is needed to approximate those terms. Since the governing equations are fairly standard they will not be presented here. The computer code used was the commercial CFD package FLUENT and the second order upwind scheme was employed.

The current CFD study has been carried out on a NACA $64_{1}-412$ half wing aspect ratio of 8.2 used in the experimental study by Render et al. ${ }^{11}$ at a Reynolds number of $5.5 \times 10^{5}$. The wing had a chord of $197 \mathrm{~mm}$ and the span was $812 \mathrm{~mm}$, which gave a true aspect ratio of 8.24 . The damage hole had a diameter of $0.2 \%$ chord and was located at half chord for three different spanwise locations. This paper will consider two of these locations. $450 \mathrm{~mm}$ and $650 \mathrm{~mm}$ from the wing root. The first of these is termed mid-span and was located at 0.55 of semi-span and the second is called the tip (located at 0.8 semi-span).

An unstructured grid was employed as it was easier to define the mesh for the damage hole. Figure 1 shows the surface mesh of the wing generated using Gambit. The baseline grid had 395,000 cells and a grid refinement study ${ }^{12}$ was conducted using a finer grid of 690,000 cells with little difference (maximum $\mathrm{C}_{\mathrm{p}}$ difference less than $5 \%$ ) between results obtained by those two grids. Hence 690,000 cells were regarded as sufficient for the current study. The Spalart-Allmaras turbulence model was used, because previous studies ${ }^{12}$ on two-dimensional battle-damaged airfoils had shown that this model produced reasonable results, and outperformed the $\mathrm{k}-\varepsilon$ model slightly.

A uniform velocity was specified at inlet and a zero gradient outflow boundary was applied downstream at the outlet. A solid wall boundary condition was applied on the wing surface and inside the hole. A stress free boundary condition was applied in the vertical direction while in the spanwise direction solid wall boundary condition was applied. Since the experiment $^{11}$ was conducted in a low speed, low turbulence $(0.1 \%$ turbulence intensity) wind tunnel the turbulent eddy viscosity at inlet required by the SpalartAllmaras turbulence model was specified

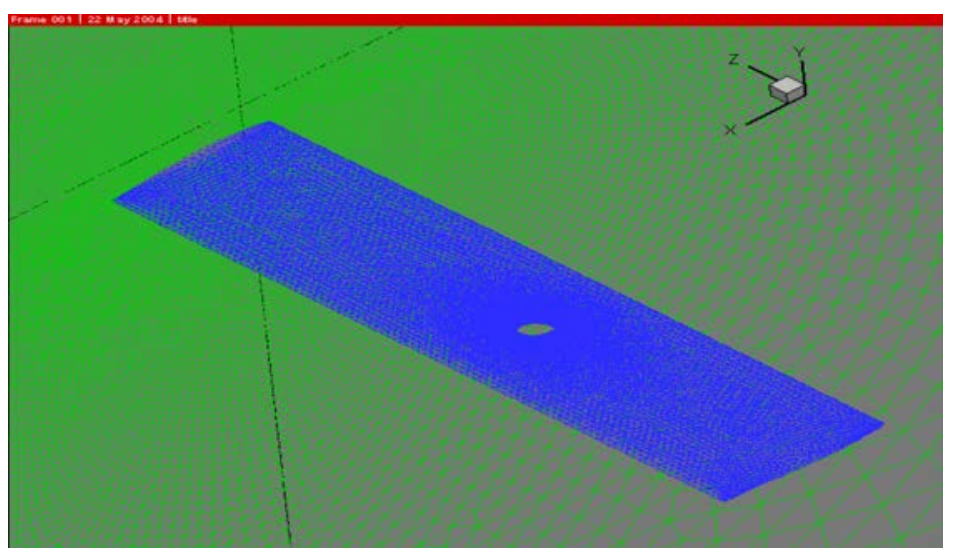

Figure 1. Wing geometry and surface mesh. to be equal to molecular viscosity.

\section{Results and Analysis}

\section{A. Validation of CFD Arrangement}

CFD results were first validated against measured aerodynamics coefficients for an undamaged wing (the same aerofoil profile and aspect ratio) to make sure that the CFD setup was acceptable. The comparison between the predictions and the measured $\mathrm{C}_{\mathrm{L}}$, and $\mathrm{C}_{\mathrm{D}}{ }^{12}$ is shown in Fig. 2 and it can be seen that there was good agreement between the predicted $C_{L}$ and the measured one. The predicted $C_{D}$ agreed reasonably well with the measured one, with slightly higher values predicted at all incidences. 


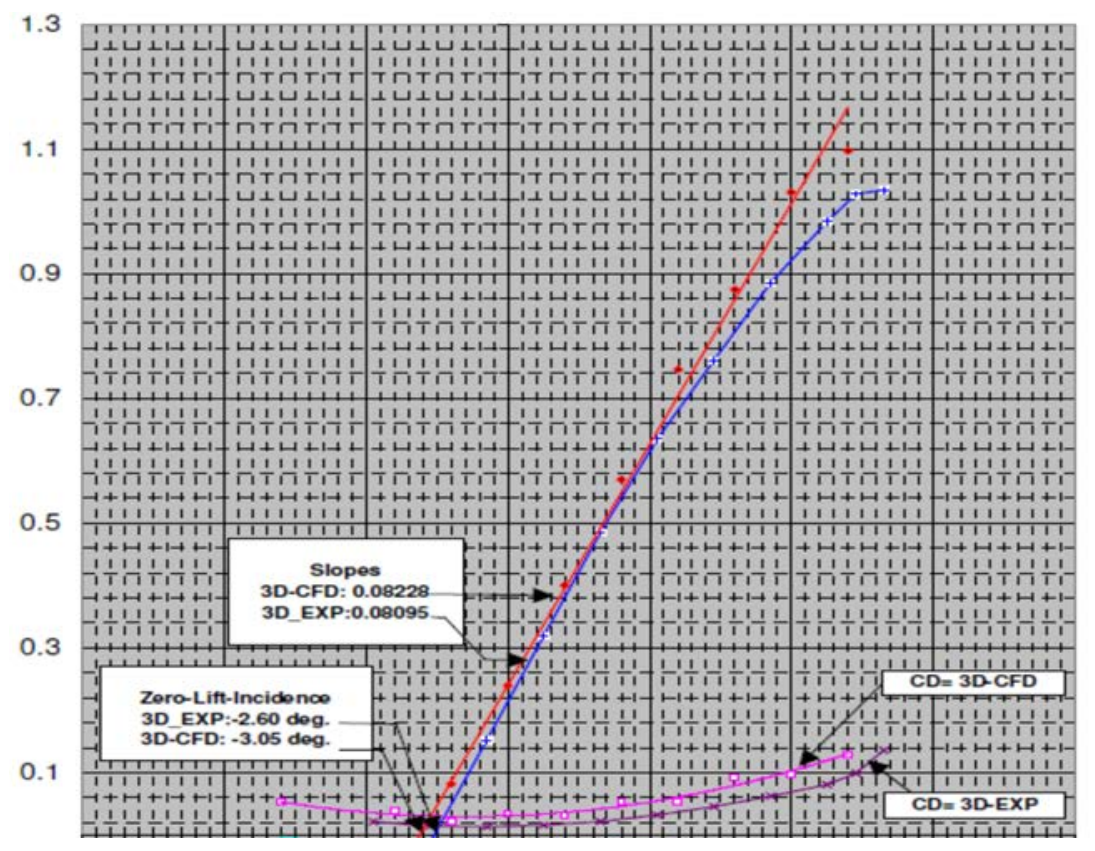

Figure 2. Lift and drag coefficient distributions for the undamaged wing.

\section{B. Damage Jet}

The influence of battle damage on the aerodynamic coefficients is due to air flow through the hole driven by the pressure differential between the upper and lower wing surfaces. This flow is similar to "jet in crossflow" and may take one of two forms. The first is a weak-jet (Fig. 3) where the flow through the hole forms an attached wake on the airfoil's surface. This causes relatively small changes in force and moment coefficients, and results in a limited disruption of the pressure distribution on the surface of the wing. The second is a strong jet (Fig. 4) formed due to increased pressure differential between the upper and lower wing surfaces when incidence is increased. In the strong jet case the flow through the hole penetrates into the freestream resulting in separation of the oncoming surface flow, and the development of a separated wake with reverse flow. Compared to the weak jet, the effect on force and moment coefficients is increased, and the influence on the airfoil pressure distribution extended significantly in a spanwise direction.

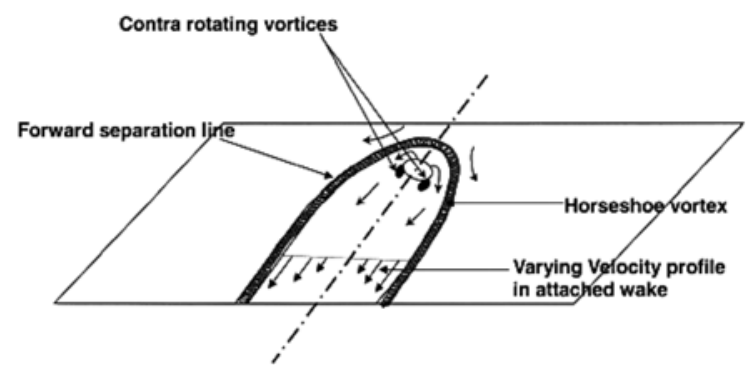

Figure 3. Weak jet flow characteristics

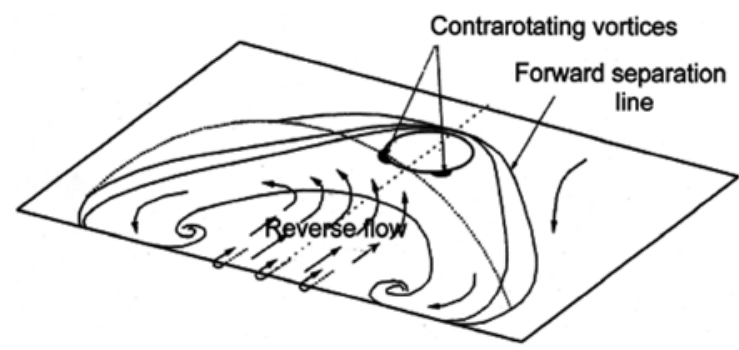

Figure 4. Strong jet flow characteristics 


\section{Flow Visualisation}

Figure 5 shows the predicted stream-traces (fluid particle path lines) of the upper-surface flows for the tip damage at three incidences of $4^{0}, 8^{0}$ and $10^{\circ}$. The flow direction is from top right-hand corner to bottom left-hand corner, with the tip of the wing at the bottom. This case is shown because any spanwise effects on the battle damage flow are likely to be most significant near the tip. Figure 6 shows the predicted stream-traces in the $x-y$ plane across the centre of the damage hole at $4^{0}, 8^{0}$ and $10^{0}$ incidences. A horseshoe vortex is clearly shown at $4^{0}$ incidence (Fig 5a). However, immediately downstream of the hole, there is a region of reverse flow. Figure 6a shows that this flow is entrained into the damage jet. However, the jet does not penetrate significantly into the freestream flow, and the flow is attached to the airfoil at around $75 \%$ chord. This is a transitional jet, whose characteristics combine those of a weak and strong jet.

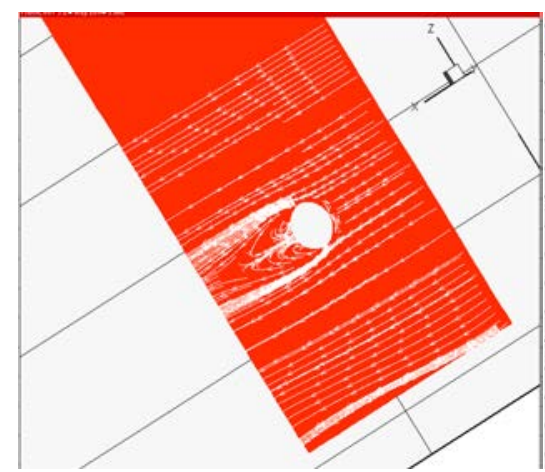

a)

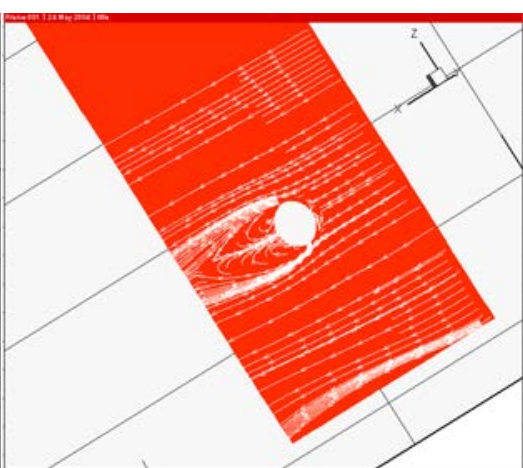

b)

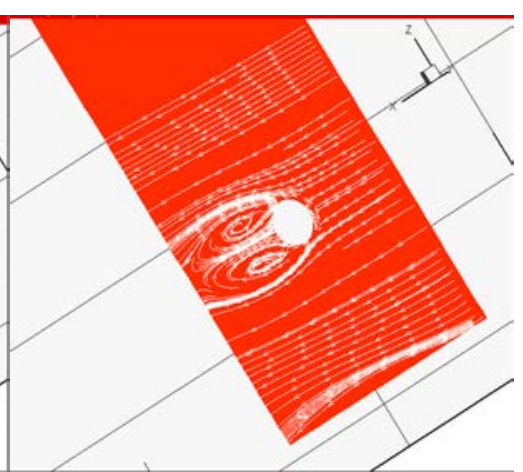

c)

Figure 5. Stream-traces of the upper-surface flows at incidences of $4^{0}(\mathrm{a}), 8^{0}$ (b) and $10^{0}$ (c) for the tip location; (Flow direction is from right to left; outboard side is at the bottom).

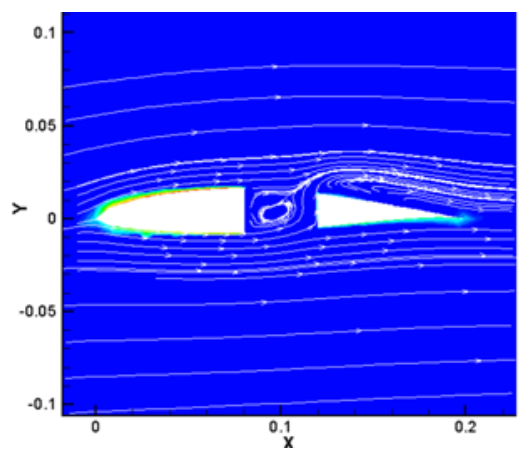

a)

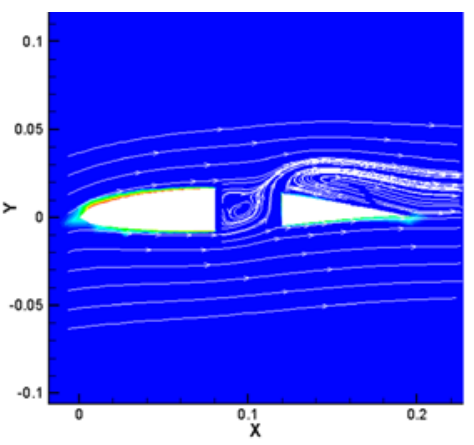

b)

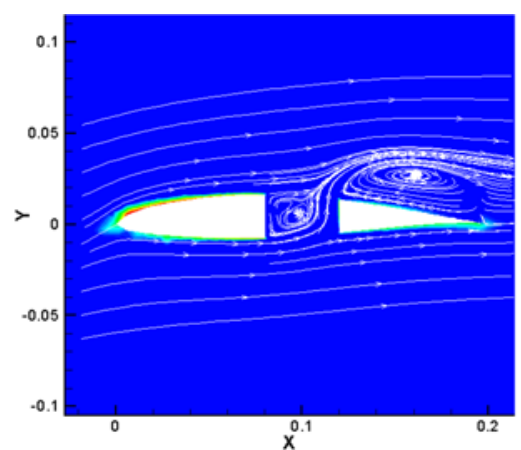

c)

Figure 6. Stream-traces of the flows in the $x-y$ plane across the centre of the damage hole at the tip location for incidences of $4^{0}$ (a), $8^{0}$ (b)and $10^{0}$ (c); (Flow direction is from right to left).

When the incidence of the wing was increased to $8^{0}$, the strength of the damage jet increased and the extent of the reverse flow behind the hole increased. This reverse flow is formed between two contra rotating vortices seen on the surface of the airfoil. It was not until $10^{\circ}$ incidence that all of the flow features associated with a strong jet were apparent. A big separation bubble forms downstream the damage hole with a very strong reverse flow, and the flow does not reattach till the trailing edge. Another feature of strong jet flow is the pair of contra rotating vortices seen on the surface of the airfoil (Fig 5c), which appear to be more defined than at lower incidences. From the positions of the forward separation lines in Fig 5 it is clear that the damage jet exits from the rear of the hole. This is confirmed by the stream-traces in Fig 6. Another interesting feature is that the flow is slightly asymmetric, especially the wake behind the damage hole as can be seen particularly in Fig.5c.

Figure.7 shows flow-visualization photographs for the damage hole. These photographs show the upper-surface flows (the flow direction is from top to bottom) at incidences of $4^{0}, 8^{0}$ and $10^{0}$. The tip is on the left-hand edge of the photographs, opposite of the tip location shown in Fig.5. The predicted main flow features shown in Figs.5 and 6 can also be observed in Fig. 7 although there are some discrepancies. In the experiment at $4^{0}$ incidence the damage 
jet was weak, with the wake fully attached downstream of the hole. At $8^{0}$ incidence the CFD results indicate that the damage jet is in a "transition" state with features of a weak jet still present whereas the flow-visualization photograph show all characteristics of a strong jet. The dark band running from the trailing edge to the hole in Fig $5 \mathrm{~b}$ is the region of reverse flow between the contra rotating vortices. The asymmetric nature of the damage flow observed in the CFD prediction is more clearly shown in these flow-visualization photographs, confirming that the asymmetry observed is a genuine effect and is likely caused by the spanwise variation of static pressure associated with finite-aspect-ratio wings. This asymmetry is also evident in Fig.8 which shows the predicted $\mathrm{C}_{\mathrm{p}}$ distributions at two spanwise locations $60 \mathrm{~mm}$ from either side of the damage hole centre, i.e., the inboard location is $590 \mathrm{~mm}$ from the wing root ( 0.726 of semi-span) and the outboard location is $710 \mathrm{~mm}$ ( 0.874 of semi-span). As can be seen clearly in Fig. 8 there is a difference between $C_{p}$ values at these two locations, more so for $8^{0}$ incidence. From the experiments the positions of the forward separation lines relative to the hole indicate that the damage jet did not occupy all of the hole. This too was confirmed by CFD.

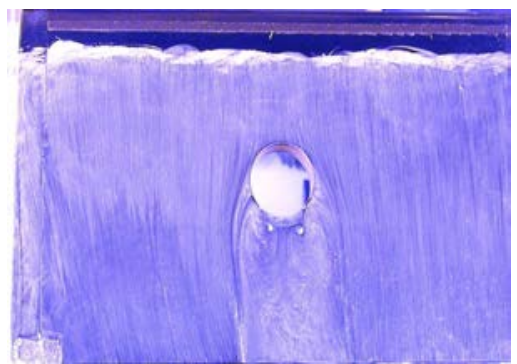

a)

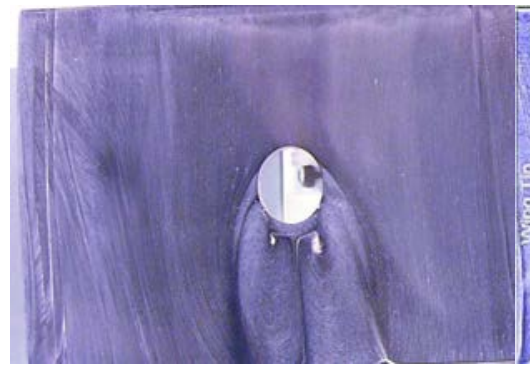

b)

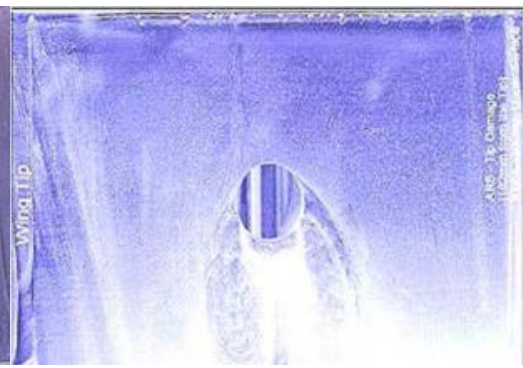

c)

Figure 7. Flow-visualization of the upper-surface flows at the tip location for incidences of $4^{0}$ (a), $8^{0}$ (b) and $10^{\circ}$ (c); (flow direction is from top to bottom; outboard side is on the left).

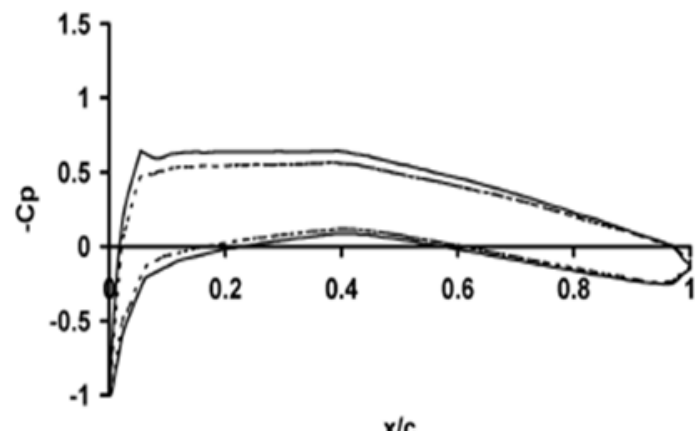

$\mathrm{x} / \mathrm{c}$

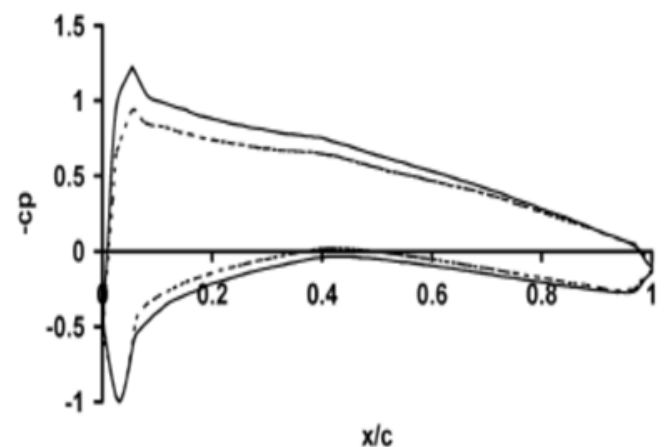

$x / c$

a)

b)

Figure 8. Predicted pressure coefficient distributions at incidences of $4^{0}$ (a), $8^{0}$ (b);(dashed line: outboard $710 \mathrm{~mm}$, solid line: inboard $590 \mathrm{~mm}$ ).

\section{Surface Pressure Coefficients}

The comparison between the predicted and measured $\mathrm{C}_{\mathrm{p}}$ profiles along the hole centerline at the tip location and at $8^{0}$ incidence is shown in Fig. 9. The CFD results show $C_{p}$ values on both upper and bottom surfaces while the experimental data are only available for the upper surface. It can be seen from the figure that the agreement between the prediction and experimental data before the damage hole is quite good although the measured peak value is higher. However, the predicted profile is different from the measured one after the damage hole and the CFD results show a peak close to the hole while the measured peak is much further downstream. This is not unexpected since the CFD flow visualization results show that the damage jet is still in a "transition" state while the experimental photographs suggest a strong jet, with the contra rotating vortices centred further downstream of the hole than in the CFD. This accounts for the different positions in the pressure peaks. For the experiment the reverse flow accelerates 
from the trailing edge of the airfoil before slowing down as it approaches the damage jet. For the CFD the acceleration happens much closer to the hole.

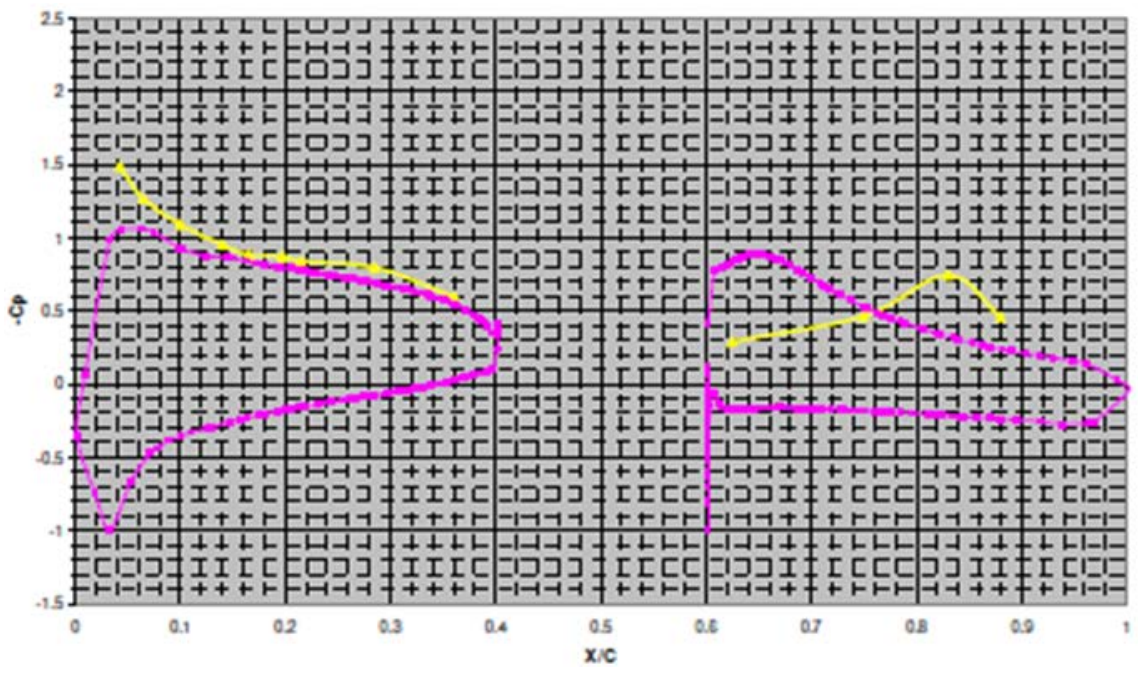

Figure 9. Pressure coefficient distributions along the hole centerline at $8^{0}$ incidence (pink curve - CFD, yellow curve - Experiment.).

\section{E. Flow structures downstream and in hole}

As shown in Figs. 2 and 9 the CFD predictions compared reasonably well with the experimental data and hence detailed study of the flow field inside the damage hole and in the region downstream of the hole were carried out using CFD. Detailed experimental measurement are difficult to carry out and are not currently available. Results are presented for the mid-span case at $8^{0}$ incidence. To allow comparison with the tip case the stream-traces on the upper surface are shown in Fig 10 and the stream traces in the x-y plane on the hole centre line are shown in Fig 11. Flow visualisation from the experiment is shown in Fig 12.

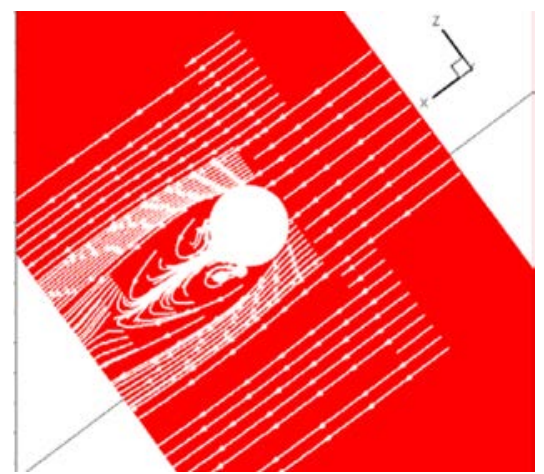

Figure 10. Stream-traces on upper surface. Midspan location. $8^{0}$ incidence. Flow direction from top right to bottom left

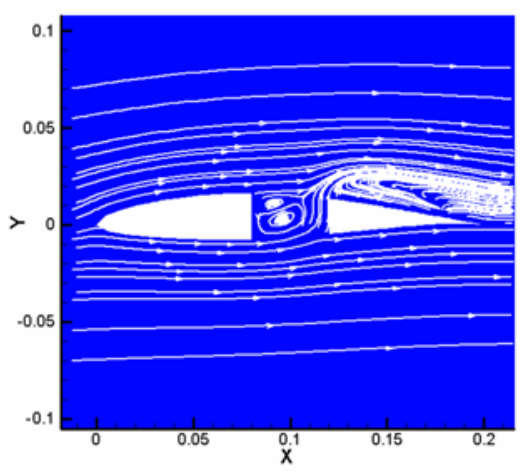

Figure 11. Stream-traces in the $x-y$ plane on hole centre line. $8^{0}$ incidence. Mid-span location. Flow from left to right.

The flows in Figs 10 and 11 are similar to those seen in Figs $5 \mathrm{~b}$ and $6 \mathrm{~b}$, suggesting that the damage jet is transitional and hasn't quite become a strong jet. As with the tip, the experiments indicated a strong jet was present at this incidence (Fig 12). The flow inside the hole is very complicated, not like a usual jet flowing through a nozzle, as can be seen in Fig. 11. There is a large counter clockwise recirculation region inside the hole, which is present for all of the cases considered and the flow exits from the rear part of the hole. For the mid span case there 
is clear evidence of a second rotating region in the upper forward part of the hole. The rotation is clockwise. This forward part of the hole can be likened to an open cavity flow.

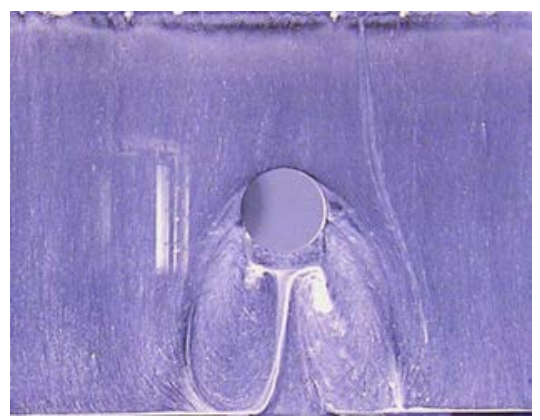

\section{Figure 12. Flow visualization on upper surface for mid-span case. $8^{0}$ incidence. Flow from top to bottom}

Figure 13 shows the stream-traces on the surface of the airfoil immediately downstream of the hole. Although not particularly evident in Fig 10, some asymmetry can be seen in the main flow features. These can be attributed to finite aspect ratio effects. The previously identified areas of recirculation are a pair of contra rotating vortices. The origin of these vortices can be seen in Fig 14. This figure shows detailed stream-traces near the downstream wall of the hole. This confirms that the flow goes out from the rear part of the damage hole and that in this region the flow field is relatively simple. Figure 14 also reveals the presence of a vortex close to the lateral/side wall of the hole and forward of the jet. This vortex feeds flow into the contra rotating vortices seen on the upper surface of the airfoil.

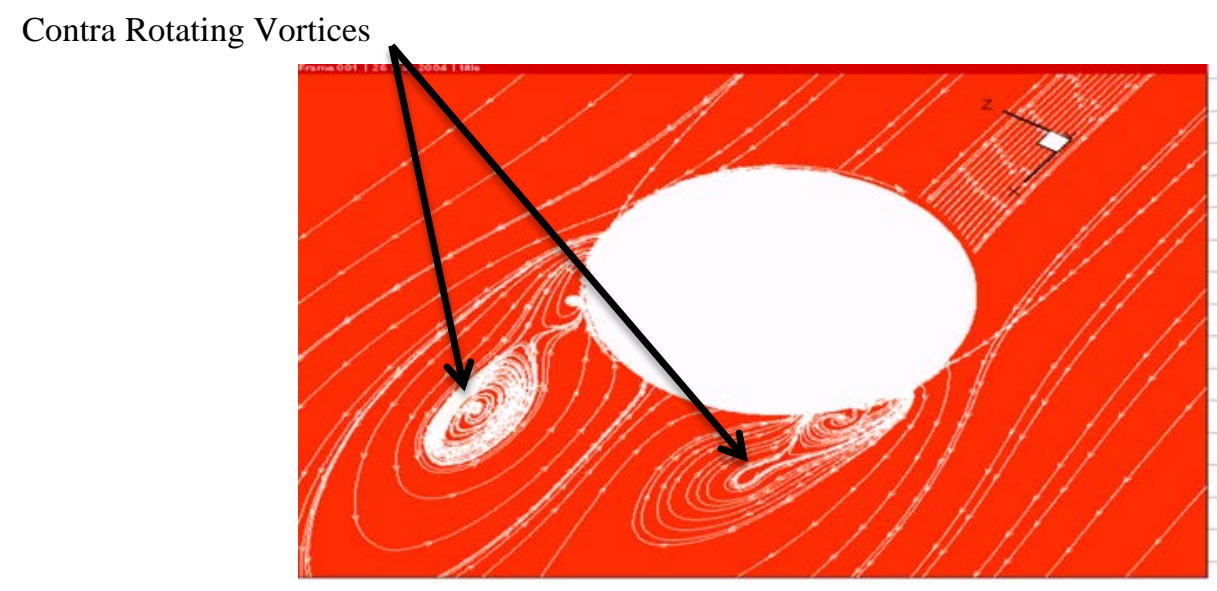

Figure 13. Stream-traces around the damage hole showing the contra-rotating vortices 


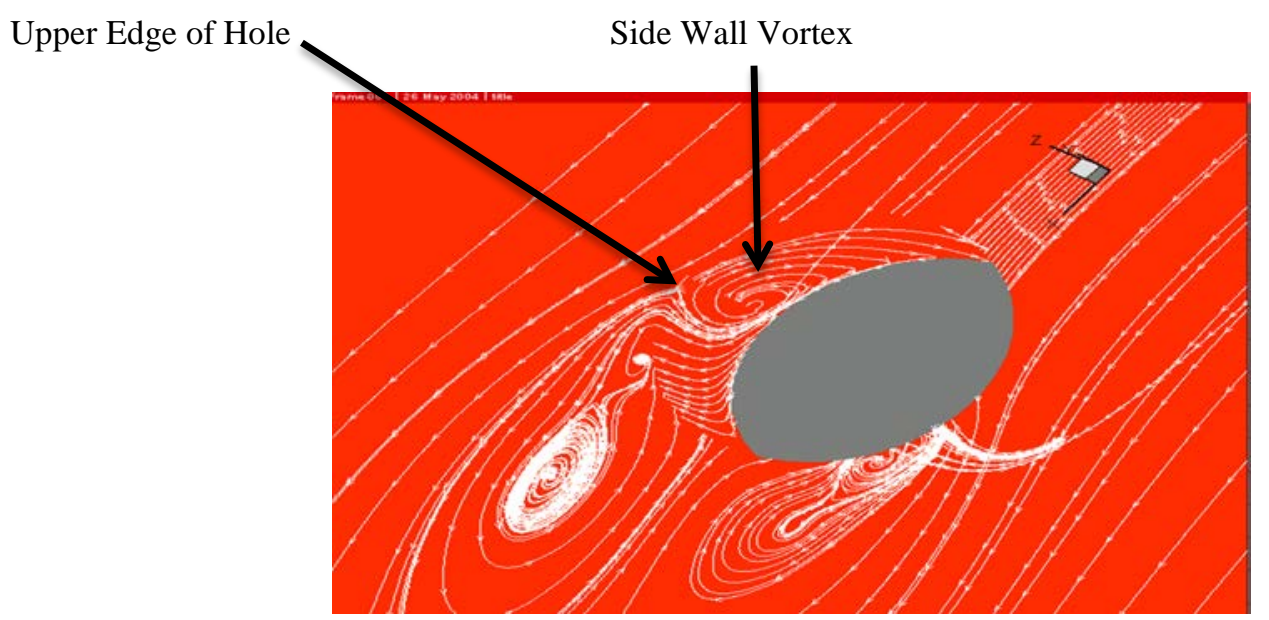

Figure 14. Stream-traces inside/around the damage hole showing the side wall vortex.

Figure 15 shows the stream traces on the upstream wall of the hole. The sidewall vortices can be seen on both sides of the hole with the air moving down towards the lower surface of the airfoil. Immediately above the side wall vortices the flow is moving upwards and appears to form a small region of upstream reverse flow on the airfoil surface immediately in front of the hole. This reverse flow is then turned into the freestream direction to form the forward upper recirculating area seen in Fig 11.

It is evident from the above that the flow field inside and around the damage hole is very complicated and several new flow features such as the side wall vortex and the upstream reverse flow region very close to the damage hole edge have been identified from the CFD results.

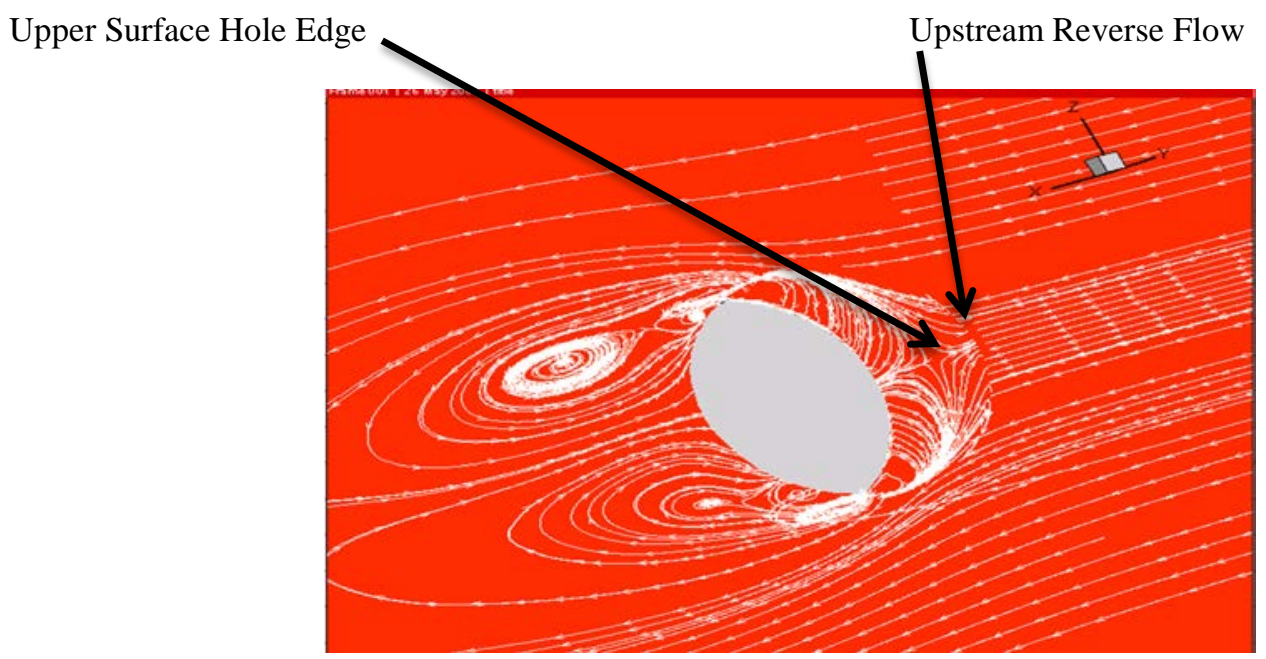

Figure 15. Stream-traces inside/around the damage hole showing the upstream reverse flow region. 


\section{Conclusions}

A CFD study has been carried out on a battle damaged NACA $64_{1}-412$ half wing of aspect ratio 8.2 at a Reynolds number of $5.5 \times 10^{5}$ and the numerical predictions compare reasonably well with the experimental data qualitatively and quantitatively. On finite aspect ratio wings, CFD results have confirmed the previous experimental finding that flows through simulated gunfire damage are asymmetric, i.e. differences exist when comparing inboard and outboard sides of the damage. This asymmetry is driven by the spanwise variation of static pressure associated with finite aspect ratio wings. However, at $8^{0}$ incidence the CFD results indicate that the damage jet is in a "transition" state with features of a weak jet still present whereas the experiment shows all the characteristics of a strong jet. This is the most likely reason why there is a big discrepancy between the predicted $\mathrm{Cp}$ values and the experimental data downstream of the hole at this incidence. Further CFD studies with more advanced turbulence models such as a Reynolds stress transport model may help to improve the accuracy of prediction. Nevertheless the current CFD study with a simple turbulence model has produced reasonably good results and especially has shown some detailed flow field inside/around the damage hole identifying a few new flow features such as the side wall vortex.

\section{References}

1. Hayes, C., "Effects of Simulated Wing Damage on the Aerodynamic Characteristics of a Swept Wing Airplane Model,” NASA TMX-1550, 1968.

2. Spearman, M.L. and Blair, A.B., "Wind Tunnel Studies of Simulated Damage to Aerodynamic Surfaces of Airplanes,” NASA TMX-2550, 1972.

3. Betzin, M. and Brown, D.H., "Aerodynamic Characteristics of an A-4B Aircraft with Simulated and Actual Gunfire Damage to on Wing,” NASA TMX-73119, 1976.

4. Spearman, M.L., "Wind Tunnel Studies on the Effects of Simulated Damage on the Aerodynamic Characteristics of Airplanes and Missiles," NASA TM-84588, 1982.

5. Irwin, A.J., "Investigation into the Aerodynamic Effects of Simulated Battle Damage to a Wing," Ph.D Thesis, Loughborough Univ., Loughborough, England, U.K., May 1999.

6. Irwin, A.J. and Render P.M., "The Influence of Mid-Chord Damage on the Aerodynamic Characteristics of Two-Dimensional Wings,” The Aeronautical Journal Vol. 104, No. 1033, 2000, pp. 153-161.

7. Schemenky, R. and Howell, G., "Aerodynamic Accounting Technique for Determining Effects of Nuclear Damage to Aircraft, Volume I - Empirical Methods,” Report No. DNA 4531F-1, 28 Feb. 1978. Prepared by General Dynamics Corporation, Fort Worth Division, P.O. Box 748, Fort Worth, Texas, 76101.

8. Rasi Marzabadi, F., Ajalli, F. and Mani, M., "Numerical Aerodynamic Analysis of a Damaged Airfoil," AIAA-2007-4180, 25th AIAA Applied Aerodynamics Conference, Miami, USA, 2007.

9. Rasi Marzabadi, F., Ajalli, F. and Mani, M., "Numerical Aerodynamic Analysis of a Damaged Airfoil with Wall Effects," HEFAT2007-SM5, 5th International Conference on Heat Transfer, Fluid Mechanics and Thermodynamics, Sun City, South Africa, 2007.

10. Saeedi, M., Ajalli, F. and Mani, M., "A Comprehensive Numerical Study of Battle Damage and Repairs upon the Aerodynamic Characteristics of an Airfoil,” The Aeronautical Journal, Vol. 114, No. 1158, 2010, pp. 469-484.

11. Render, P.M., Samad-Suhaeb, M., Yang, Z. and Mani, M., "Aerodynamics of Battle-Damaged FiniteAspect-Ratio Wings,” Journal of Aircraft, Vol. 46, No. 3, 2009, pp. 997-1004.

12. Samad-Suhaeb, M., "Aerodynamics of Battle Damaged Finite Aspect Ratio Wings," Ph.D. Thesis, Loughborough Univ., Loughborough, England, U.K., Apr. 2008. 